\title{
Entre Ulysse et Robinson
}

L'exode vu par Alfred Döblin (été 1940)

Döblins Schicksalsreise als moderne Odyssee und neue Robinsonade

Between Ulysses and Robinson: the Exodus Seen by Alfred Döblin (Summer 1940)

\section{Françoise Knopper}

\section{(2) OpenEdition}

\section{Journals}

Édition électronique

URL : http://journals.openedition.org/ceg/4865

DOI : $10.4000 /$ ceg. 4865

ISSN : 2605-8359

\section{Éditeur}

Presses Universitaires de Provence

\section{Édition imprimée}

Date de publication : 2 mai 2019

Pagination : 203-214

ISBN : 979-10-320-0214-8

ISSN : 0751-4239

\section{Référence électronique}

Françoise Knopper, «Entre Ulysse et Robinson », Cahiers d'Études Germaniques [En ligne], 76| 2019, mis en ligne le 11 novembre 2020, consulté le 25 janvier 2021. URL : http://journals.openedition.org/ ceg/4865; DOI : https://doi.org/10.4000/ceg.4865 


\title{
Entre Ulysse et Robinson \\ L'exode vu par Alfred Döblin (été 1940)
}

Françoise KNOPPER

Université Toulouse-Jean Jaurès, CREG (EA 4151)

\begin{abstract}
Alfred Döblin, médecin et célèbre romancier qui s'était réfugié dès 1933 à Paris, a relaté dans Schicksalsreise ${ }^{1}$ l'exode au cours duquel il traversa la France entre les mois de mai et de septembre 1940, avant de passer par l'Espagne et gagner Lisbonne. La trame factuelle de ce livre autobiographique ne se limite d'ailleurs pas à cette partie de l'exil car Döblin relate aussi son installation aux États-Unis puis son retour en Allemagne en $1946^{2}$. Ce texte peut ainsi s'inscrire dans la longue liste de ces témoignages individuels qui font partie intégrante de l'historiographie des migrations, de ces relations d'exilés et de migrants qui continuent à être collectées et archivées ${ }^{3}$. L'utilisation réfléchie et méthodique de ces écrits dits du for privé est dorénavant préconisée par beaucoup d'historiens et, corollairement, les théoriciens de la narratologie attirent l'attention sur les différences des traditions discursives et des logiques de la littérature et de l'histoire ${ }^{4}$. Par ailleurs, les méthodologies sont à ajuster en fonction du support textuel. Nous pouvons le constater en mettant par exemple en perspective les relations que deux exilés ont faites de leur périple en
\end{abstract}

1. Alfred Döblin, Schicksalsreise. Bericht und Bekenntnis, Frankfurt a. M., Verlag Josef Knecht, 1949, 479 p. L'édition utilisée ici est : Schicksalsreise. Bericht und Bekenntnis. In Verbindung mit den Söhnen des Dichters éd. par Anthony W. Riley, München, Deutscher Taschenbuch Verlag, 1996 (les pages citées figureront entre parenthèses). Le texte a été traduit en français par Pierre Gallissaires, Voyage et destin. Récit et confession, Monaco/ [Paris], Éd. du Rocher, 2002.

2. Christine Maillard, Monique Mombert (dir.), Der Grenzgänger Alfred Döblin, 1940-1957, Biographie und Werk, Internationales Alfred-Döblin-Kolloquium Strasbourg 2003, Jahrbuch für internationale Germanistik, A, t. 75, 2003. Et ici « "Ich kannte die Deutschen”. Alfred Döblins Auseinandersetzung mit Nationalsozialismus und Holocaust », p. 187-197.

3. Après un premier travail mené par Jean Vidalenc (L'Exode de mai-juin 1940. Publié par le Comité d'histoire de la Deuxième Guerre mondiale, Paris, PUF, 1957), dans lequel Döblin est l'un des auteurs cités, il a fallu attendre plusieurs années pour que les témoignages sur l'exode de 1940 soient à nouveau recherchés ou exhumés. Cf. à ce sujet en particulier Pierre Miquel, L'Exode. 10 mai20 juin 1940, Paris, Le Grand Livre du Mois, 2003, et Éric Alary, L’Exode. Un drame oublié, éd. augmentée, Paris, Perrin, 2013.

4. Notamment Wilfried Schulze, Ego-Dokumente - Annäherung an den Menschen in der Geschichte, Berlin, Akademie Verlag, 1996; Christophe Prochasson, « Les mots pour le dire : Jean-Norton Cru, du témoignage à l'histoire ", Revue d'histoire moderne et contemporaine 48-4, 4/ 2001, p. 160189. Un bilan des différences de traditions discursives est aussi proposé par Paul Aron dans «Écrire l'exode. Mai 40 vu par les écrivains belges », in Stefan Martens et Steffen Prauser (dir.), La Guerre de 1940. Se battre, subir, se souvenir, Villeneuve d'Ascq, Presses universitaires du Septentrion, 2014, p. 211-222. 
France entre leur départ de Paris, après le 10 mai 1940, et leur embarquement pour les États-Unis. L'un est l'écrivain Alfred Döblin, l'autre est Friedrich Stampfer, responsable politique et journaliste au Vorwärts. Bien que les expériences et le trajet offrent beaucoup de ressemblances, une analyse des souvenirs qu'ils ont publiés requiert des approches distinctes. Stampfer ${ }^{5}$ insiste sur le déplacement groupé des membres de la $\mathrm{SOPADE}$, sur les règlements et les mesures à prendre, sur l'identification des institutions administratives ou caritatives; il fait alterner la première personne du singulier et le nous collectif, ce dont on peut déduire qu'il ne perdit jamais de vue son devoir de rédiger une chronique permettant aux générations futures d'écrire l'histoire de l'an 1940. Döblin, en revanche, même quand il décrit le déplacement groupé des fonctionnaires du ministère dont il fit partie, ne se présente pas en historiographe, ne propose pas de vue d'ensemble, et son choix de ne pas élaguer l'anecdotique est le miroir de son désarroi et de sa quête de sens.

Chez Döblin, le récit des souffrances, des épreuves, des errances, s'apparente à une Odyssée littérarisée et à une robinsonnade modernisée. Mais la distance critique que le narrateur a observée envers soi-même et les autres, la précision de ses descriptions du chaos lors des évacuations de civils, l'introspection exigeante, le souci de vérité qui le caractérisent, nous incitent à examiner comment les deux modèles du genre, l'Odyssée et Robinson Crusoe, sont réinterprétés dans Schicksalsreise. C'est un texte qui illustre la complexité des liens que Döblin a établis entre histoire et littérature et la singularité d'une écriture dont Michel Vanoosthuyse a éclairé l'unicité .

Le thème de l'exil est omniprésent dans l'œuvre de Döblin, ce dernier ayant très tôt abordé la « dialectique de l'exode » dans sa dimension métaphysique sécularisée et psychiatrique, celle de la faute originelle assumée par le fils ${ }^{7}$. De plus, il avait traité des thèmes tels que la solitude de l'individu, sa désorientation, le destin de la collectivité juive, la lutte pour la survie, aussi bien dans sa prose fictionnelle que dans la relation de l'un de ses rares voyages, celui qu'il avait entrepris depuis Berlin pour remonter jusqu'au noyau originel et vivant de la culture juive en Pologne (Reise in Polen). L'exil qu'il a vécu entre 1940 et 1946 a concrétisé ses anciennes préoccupations, celles d'un exil qui était jusqu'alors resté pour ainsi dire fantasmatique, et a fait atteindre à sa quête de sens un point de non-retour.

Les étapes de la rédaction de cet ouvrage peuvent se retracer à partir des manuscrits conservés à Marbach. Döblin a tenu un journal durant son exil et a ensuite travaillé à partir de ces notes; entre octobre 1940 et janvier 1941, à Hollywood, il a écrit un texte dont le titre est « Robinson in Frankreich - Erlebtes. Mai-September 1940 ».

5. Friedrich Stampfer, Erfahrungen und Erkenntnisse. Aufzeichnungen aus meinem Leben, Köln, Verlag für Politik u. Wirtschaft, 1957.

6. Sur la subversion des divers codes narratifs et le rejet du dogme esthétique par Döblin, cf. les travaux que Michel Vanoosthuyse a consacrés à cet auteur, notamment Alfred Döblin. Théorie et pratique de "l'œuvre épique », Paris, Belin, 2005, en particulier le chapitre " L'exil, la révolution, le Christ » (p. 127-189). Du même : Petite introduction à l'œeuvre de Döblin. Du döblinisme, conférence du mercredi 28 août 2013 [Date de rédaction 10 décembre 2009], URL : ethttp://www.alfred-doblin. $\mathrm{com} /$ portrait/doblinisme-introduction-oeuvre-de-doblin/ [dernière consultation le 27 février 2018].

7. Louis Huguet, L'œuvre d'Alfred Doeblin ou la Dialectique de l'exode. Essai de psycho-critique structurelle, repr. des thèses Lille III, 1978, p. 613-614. 
Mais le livre ne sera publié qu'en 1949, dans une seconde version que Döblin a reprise et complétée en 1948, à l'époque où il faisait partie des forces françaises d'occupation. Le titre en est devenu : Schicksalsreise; le sous-titre de cette seconde version, Bericht und Bekenntnis, se place sous le signe de la conversion de Döblin, qui, tel Claudel en 1886, rapporte avoir vécu une expérience mystique devant le crucifix de la cathédrale de Mende. La longue introspection qu'il mène dans Voyage et destin se conclut par une phrase significative de la radicalité de la conversion identitaire que cette forme d'anamnèse aurait entraînée pour lui : « Eine schwere Maske fällt von ihm. Er sieht, daß ihn eine dicke Tonmaske preßte und sich für sein Ich ausgab » (p. 367).

De ce fait, bien que Schicksalsreise ne soit pas le seul témoignage que l'auteur a donné de son exil, c'est le texte où la signification de l'exil se trouve potentialisée ${ }^{8}$, comme ritualisée, et l'écriture parfois sacralisée : «Die Reise verlief an mir, mit mir und über mir. Nur weil es sich so verhielt, begebe ich mich daran, die Fahrt, ihre Umstände, aufzuzeichnen (p. 65). »

Cet apport mystique ne faisait pas l'objet de l'article Abschied und Wiederkehr que Döblin avait publié en 1946 dans plusieurs journaux - d'abord dans la Badische Zeitung $^{9}$ en février - et où il synthétisait les informations sur son exil à l'étranger puis ses impressions à son retour en Allemagne. L'absence de connotations religieuses dans cet article est d'autant plus frappante que des passages en seront repris trois ans plus tard, souvent littéralement, dans Schicksalsreise ${ }^{10}$.

Le fait que ce soient deux mythes canoniques, celui d'Ulysse et celui de Robinson, qui sous-tendent le témoignage d'un exilé, ne paraît pas, en soi, exceptionnel ${ }^{11}$, mais, sous la plume de Döblin, leurs ressources sont à la fois exploitées et subverties. D'une part, comme chez d'autres auteurs ${ }^{12}$, narration d'un périple et mythographie se recoupent aisément : en effet, comme ce genre de relation est nécessairement fragmenté et tributaire de la discontinuité de la mobilité, il est fréquent que l'Odyssée ou la robinsonnade fournissent des modèles, des schémas narratifs sur lesquels s'appuyer; le mythe se définit alors comme une forme archétypique qui aide à structurer et organiser le parcours. D'autre part, dans Schicksalsreise,

8. Michel Vanoosthuyse, «L'exode et le royaume. Schicksalsreise d'Alfred Döblin », in Marx et autres exilés. Études en l'honneur de Jacques Grandjonc, réunies par Karl Heinz Götze, Cahiers d'Études Germaniques 42 /2, 2002, p. 267-279.

9. Ce même article paraît encore le $1^{\text {er }}$ mai 1946 dans la revue La otra Alemania, t. 8, nº 117, p. 6-7.

10. Un long extrait en est repris dans le chapitre «Europa » (Schicksalsreise, p. 305-309). Sur la spécificité de cet apport mystique dans la littérature de l'exil, cf. l'article de Frederik Offen, « Döblins Schicksalsreise », et la bibliographie correspondante, in Handbuch der deutschsprachigen Exilliteratur. Von Heinrich Heine bis Herta Müller, éd. par Bettina Banasch, Gerhild Rochus, Berlin, de Gruyter, 2013, p. 277-284, avec une bibliographie sélective afférente.

11. Wolfgang Frühwald, «Odysseus wird leben. Zu einem leitenden Thema in der deutschen Literatur des Exils 1933-1945 », in Werner Link (éd.), Schriftsteller und Politik in Deutschland, Düsseldorf, Droste, 1979, p. 100-1I3 ; Renate Schlesier, « Transgressionen des Odysseus », in Renate Schlesier, Ulrike Zellmann (éd.), Reisen über Grenzen. Kontakt und Konfrontation, Maskerade und Mimikry, Münster, Waxmann, 2003, p. 133-142.

12. Paul Michael Lützeler, « Migration und Exil in Geschichte, Mythos und Literatur », in Handbuch der deutschsprachigen Exilliteratur, p. 3-25, en particulier p. 9-12. 
le mythe permet d'associer existence singulière et réflexion sur l'homme, texte autobiographique et révélation d'une transcendance universelle.

Enfin, si les termes et les thèmes de l'Odyssée et de Robinson apparaissent chez Döblin dans le texte édité en 1949, alors qu'ils avaient été au contraire soigneusement écartés dans celui de 1946, nous constaterons que l'Odyssée est transformée et étroitement circonscrite, si bien que le modèle grec est abandonné. Il s'y substitue sans doute la construction d'une odyssée kierkegaardienne, car la pensée du philosophe Kierkegaard a pu jouer un rôle dans cette revisite des mythes par Döblin. Au demeurant, la référence dont Döblin se réclame plus explicitement est celle de Robinson; néanmoins, sur ce point encore, le genre et la tradition de la robinsonnade sont amputés, tronqués, remis en question.

\section{Sortie de l'Odyssée homérique}

La qualification d'odyssée à propos de périples marqués par l'imprévu et le désarroi est si fréquente sous la plume des auteurs et des commentateurs qu'elle risque de perdre son sens et de devenir une évidence stéréotypée. Héritée de la culture humaniste, l'odyssée littérarisée est commode puisqu'elle est appropriée à synthétiser à elle seule les caractéristiques de l'errance, son indétermination temporelle ou spatiale, sa succession de haltes, d'obstacles, de dangers. Et ainsi appropriée à faire partager à elle seule les émotions et les souffrances des acteurs. Cette qualification semble donc opératoire aussi pour la relation que Döblin a faite de son propre exode de 1940, pour ce temps d' « errance rocambolesque et dramatique à la fois ${ }^{13}$ » qui caractérisa les semaines que Döblin a passées entre Paris, Mende, Toulouse et Lisbonne.

Döblin était exilé depuis 1933 à Paris. Comme son environnement géographique, professionnel, social, affectif donnait un sens - même précaire - à cette première phase de son exil, il avait fait, en un premier temps, tout son possible pour préserver ces acquis. Cependant, son ami Robert Minder lui ayant fait admettre après le 10 mai 1940 qu'il fallait partir de Paris, son exil entra dans une nouvelle phase, celle de l'exode, sorte d'exil redoublé : «Flucht aus unserm Zufluchtsort » (p. 18). D'abord ce sont sa femme et son fils Stephan qu'il envoie dans le Midi et ensuite c'est avec les fonctionnaires du ministère de l'Information (et notamment Robert Minder) qu'il est convoyé vers Tours puis vers Bordeaux. Dans ce train, il garde des habitudes de touriste et, comme dans le récit de son voyage en Pologne (1926), il note ses observations sur le vif et en fait un usage humoristique. À ce stade, ses impressions de voyage permettent encore de classer, d'orienter, d'ordonner la réalité, même si le mot qui caractérise la situation d'un exilé, c'est le deuil, "Trauer », note-t-il (p. 51). En cela, l'expérience du narrateur est - au début - comparable à celle des autres exilés et elle fournit un témoignage précis de l'inadaptation des mesures d'évacuation qui avaient été prises par le pouvoir politique ${ }^{14}$.

13. Pour citer M. Vanoosthuyse, " "Un monument unique". Introduction à la tétralogie d'Alfred Döblin Novembre 1918. Une révolution allemande », Agone 2008, p. 173-200, ici 173.

14. Sur ces mesures pourtant prévues de longue date, cf. P. Miquel et E. Alary. 
Mais Döblin se départ peu à peu de sa condition de voyageur. Il voit dans la guerre une force monstrueuse qui génère de l'angoisse ( «Grauen einflößt», p. 21, 28) et qui désorganise les repères dans l'espace et dans la société. Comparable à cet autre exilé qu'était Brecht et qui déplorait l'impossibilité, en ces temps de guerre, de s'adonner à des élans lyriques, Döblin note qu'il cesse d'écrire de la fiction (« Die Feder wurde mir aus der Hand geschlagen », p. 17). Puis, ayant perdu la trace de sa femme et de son fils dont il n'a plus de nouvelles, il prend une décision déraisonnable mais qui n'avait jadis rien d'exceptionnel : il part seul à leur recherche, abandonne le convoi ministériel à Cahors, se déplace en zigzags, une de ses obsessions étant sa valise, motif emblématique de la figure du migrant. Espoirs et désillusions se succèdent puisque tout le monde ignore où sa femme s'est rendue. Il s'arrête dans un camp de réfugiés à Mende, au cœur de la Lozère, où les conditions d'hébergement sont déplorables. Puis ce seront les retrouvailles à Toulouse, l'embarquement, le séjour aux États-Unis et le retour en Allemagne.

La différence avec l'Odyssée est cependant fondamentale: Ulysse rentre à Ithaque, et même si Homère donne à penser que son héros repartira sans doute un jour, cette épopée est le récit d'un retour, alors que le texte de Döblin n'est pas le récit du retour espéré car, dans l'Allemagne d'après Hitler, la rémigration n'efface pas la mémoire de l'exil :

Als ich wiederkam, da - kam ich nicht wieder. [...] Ich sehe nicht die Straßen und Menschen, wie ich sie früher sah. Auf allen liegt wie eine Wolke, was geschehen ist und was ich mit mir trage: die düstere Pein der zwölf Jahre. Flucht nach Flucht. Manchmal schaudert's mich, manchmal muss ich wegblicken und bin bitter. (p. 306-309)

L'espace retrouvé n'apporte pas le repos que pourrait procurer un port d'attache; la désillusion déjà manifeste dans Abschied und Wiederkehr en 1946 est reprise littéralement en 1949, ce qui nuira d'ailleurs beaucoup à la diffusion de Schicksalsreise et à sa réception.

Sur le plan du discours littéraire, ce récit démontre plutôt la sortie de l'univers de l'Odyssée. Il semble que le désenchantement soit si pesant qu'il rend impossible de se contenter de se référer à des destins héroïques, comme si l'attente du retour, celle qui était au centre du chant épique d'Homère, n'était plus crédible, le retour à un statu ante quo étant voué à l'échec ${ }^{15}$.

\section{Odyssée biographique sur un mode kierkegaardien}

Intériorisant la notion d'exode, l'été 1940 servant d'école de méditation, Döblin procède à une sacralisation de l'écriture de l'exil. Il semble déléguer à la Providence divine, à laquelle il se soumet et qui serait l'instigatrice de ce voyage initiatique, la fonction auctoriale qu'il assumait d'ordinaire dans ses romans :

15. Sur le désenchantement des artistes, le dossier " Artistes plasticiens de retour d'exil en pays germanophones après 1945 » publié par Jean Mortier, Jacques Poumet dans Allemagne d'aujourd 'hui 205, juillet-septembre 2013. 
Von Anfang bis Ende hatte die Reise einen - ich möchte sagen: traumhaften, imaginären Charakter; ich meine einen nicht nur realen Charakter.

Bei der Reise von ihrem Anfang bis zu ihrem Abschluß (ist er erfolgt?) reiste ,ich“. Aber der Reisende war kein gewöhnlicher Passagier mit seinem Billet. (p. 65)

Le sens étant promis, annoncé, révélé, ce voyage n'est pas un pèlerinage résultant d'un vœu ou d'une expiation mais il conduit au cœur de la réalité première, de ce que Döblin appelle « vérité ${ }^{16}$ », effaçant la faute originelle.

Le terme d'Odyssée est à prendre dans son acception originelle, et non pas seulement au sens figuré de périple. En effet, comme chez Homère, nous trouvons chez Döblin l'antique modèle anthropologique du voyageur malgré lui, qui, ballotté au gré des événements, perd la maîtrise de l'espace et dont le destin est à la fois particulier et exemplaire. Et surtout, comme chez Homère, l'exil est organisé par une force extérieure qui donnerait leur signification cachée à des retournements de situation imprévus, comme lorsque le train qui devait aller à Clermont Ferrand amène le chroniqueur à la destination dont il rêvait, au Puy (« es war ein metaphysischer Witz, [...] es war Hohn, eine Fopperei, wie ich noch mehrere erleben sollte, in einer ganzen Serie », p. 44), ou aux caprices du calendrier, Döblin ayant décidé de partir à la recherche de sa femme précisément le jour où la situation de celle-ci devenait critique au Puy. Ne croyant pas à de simples coïncidences, Döblin place son voyage « entre ciel et terre » de sorte que son récit factuel se mue en une réflexion sur le destin de l'homme, et le voyage devient une métaphore ontologique : « Ich reiste mit leichtem Gepäck. Ich wußte früher nicht, wie leicht es war » (p. 109).

Sa soumission à une force transcendantale, apparentée à celle subie par Ulysse errant après la destruction de Troie, souffrant pour son salut et espérant vainement assurer le retour de tous ses compagnons, est sans doute marquée par l'influence de sa lecture de Kierkegaard. C'est pourquoi une autre variante de l'Odyssée est lovée au cœur du texte. Une mention est faite au titre de l'Odyssée (p. 127), à vrai dire entre deux virgules et apparemment sans répercussions considérables; elle figure là où Döblin récapitule sa formation lycéenne et au fait qu'il était classique de lire Homère. Cette information n'est pourtant pas anecdotique car, quelques pages plus loin, un passage est consacré à la tranche de vie avant l'exode. Or ce passage peut se traiter en Odyssée biographique sur un mode kierkegaardien, le retour à Ithaque étant en quelque sorte remplacé par l'illumination mystique.

Le narrateur, assis sur sa couchette dans le camp de réfugiés à Mende, fait le point et jette un regard rétrospectif sur son passé berlinois. Il évoque tour à tour son métier de médecin, sa connaissance du judaïsme et son voyage en Pologne, ses convictions politiques socialistes, sa déception face aux bonzes des partis, puis le fait que lui et ses amis ont été chassés par la « terreur ». Et il commente :

Welche Sache hatte ich zu meiner gemacht?

Bin ich gestrandet, jetzt?

Ich habe mir einen zu leichten Kahn gebaut, um über den Ozean zu fahren. Natürlich wurden die Wände eingedrückt. Der Boden erwies sich als Papier und weichte auf. Ich schleppte mich

16. Jochen Meyer (éd.), Alfred Döblin 1978-1978. Eine Ausstellung des Deutschen Literaturarchivs im Schiller-Nationalmuseum Marbach am Neckar, München, Kösel, 1978, p. 384. 
weiter, so lange die Witterung es erlaubte, dann ein Windstoß, und noch ein Windstoß, und der Kahn kippte und die Seefahrt war zu Ende. (p. 133)

Cette métaphore d'une traversée des mers et d'un naufrage rappellerait Robinson mais elle se mue plutôt en Odyssée quand le narrateur dresse le bilan de sa créativité littéraire et de son travail de romancier. Le fait de produire une œuvre littéraire ne lui aurait procuré que des apaisements passagers et le travail d'écrivain aurait consisté en une succession d'aventures sans cesse recommencées :

Eine sonderbare Sache, das Schreiben. Ich begann es nie eher, bis die Einfälle einen bestimmten Reifegrad erreicht hatten, und das war dann der Fall, wenn sie im Gewande der Sprache erschienen. Hatte ich dieses Bild, so wagte ich mich mit ihm, mit meinem Pilotenboot, aus dem Hafen heraus, und da bemerkte ich draußen bald ein Schiff, einen großen Ozeandampfer, und ihn betrat ich und fuhr aus und war in meinem Element, reiste und machte Entdeckungen, und erst nach Monaten kehrte ich von solcher großer Fahrt heim, gesättigt, und konnte wieder das Land betreten. Meine Fahrten bei geschlossener Tür führten mich nach China, Indien, Grönland, in andere Epochen, auch aus der Zeit heraus. Was für ein Leben. (p. 134)

Une telle représentation de sa vie passée nous permet de déceler des mythèmes et de comparer chaque œuvre écrite à une des stations d'Ulysse :

Und ich war befriedigt, gesättigt, bis mich wieder die Unruhe und die Leere bedrängten und der Trieb in mir erwachte, und das wuchs heran und war eine Sucht, die Sucht zu reisen, zu wandern, zu fliegen, sich zu verwandeln. (p. 134)

Cette rétrospective marine diagnostique et dissèque l'instabilité et l'insatisfaction existentielle et elle nous livre une interprétation moderne des haltes et des départs successifs d'Ulysse. Ainsi que celui d'un retour intériorisé puisque cette Odyssée biographique enclenche une attente et une extase mystique :

Er (!) wußte schon früh von dem Geheimnis, - von dem Geheimnis der Welt. Er wußte, ohne daß es ihn einer gelehrt hätte, daß es vor dem Geheimnis nur eine einzige menschliche Bewegung gibt, das Hinsinken. (p. 135)

Exceptionnellement, le « moi » cède dans cette phrase la place à un « il », ce qui est peut-être un glissement vers un être humain mythique. Le narrateur se souvient en effet d'avoir pressenti qu'il y avait un absolu, « Ursinn », il aurait toujours longé un fleuve, " étroit », celui du « secret de l'existence du monde » : et c'est à ce secret qu'il s'unirait à présent, le seul geste possible étant celui du total abandon, « das Hinsinken » (p. 135).

Ce glissement de l'historiographie à la mythographie et cette métamorphose de l'Odyssée homérique peuvent résulter d'une adaptation personnalisée de la philosophie existentialiste de Kierkegaard. Hypothèse d'autant plus vraisemblable que, dans cette même rétrospective, le narrateur dit avoir découvert à Paris « le magicien » Kierkegaard, ce qui n'a pas échappé aux commentateurs de l'ouvrage ${ }^{17}$.

Sans détailler ici les fonctions qui ont été allouées par Kierkegaard au mythe et au supra-rationnel, nous rappelons ${ }^{18}$ que leur enjeu était de « réaliser le général »

17. A. W. Riley, Schicksalsreise, p. 427.

18. À partir de "Das Gleichgewicht des Ästhetischen und Ethischen in der Entwickelung der Persönlichkeit », in [Søren Kierkegaard], Entweder-Oder. Ein Lebensfragment [1843], traduit du 
sans amputer « la singularité de l'individu », de lier le particulier à l'universel, bref de rendre possible d'écrire un mythe collectif tout en le vivant en être singulier. Et Döblin semble avoir transféré le mythe théorisé par Kierkegaard afin de structurer cette rétrospective de sa vie d'avant l'exil : il la divise en trois séquences, lesquelles, peut-être, sont l'écho des trois manières d'être au monde que Kierkegaard avait distinguées. Une manière d'être au monde est le stade appelé « esthétique », que Kierkegaard voyait marqué par la jouissance du présent et la fuite à l'égard de soi-même; ce serait la vie berlinoise, durant laquelle Döblin dit avoir privilégié le présent, au détriment du passé et du futur. Le stade dit " éthique » serait ici celui de l'écriture, quand Döblin s'engageait auprès des images et des mots qui lui servaient d'environnement social, qui l'accompagnaient dans ses voyages imaginaires, conformément à ses catégories romanesques. Le stade « religieux » est, chez Kierkegaard comme chez Döblin, celui dans lequel l'individu peut entrer dans l'absolu, dans l'invisible, au prix d'un saut, d'un acte de foi sans garantie rationnelle - ce qui lui vaudra incompréhension et solitude :

Der Mangel an Gerechtigkeit in dieser Welt beweist, dies ist nicht die einzige Welt. Der Ursinn erstreckt sich nicht auf diese eine, unseren Sinnen zugängliche Welt. Sein Wirken, auch seine Gerechtigkeit, umschließt und durchdringt die sichtbare und unsichtbare Welt. Ja, noch andere Welten, unsichtbare. Welch einfache und naheliegende Gedanken. Ich fühle mich in dem Augenblick, wo ich dies denke, nicht mehr so getrieben. (p. 135)

Le saut vers une conception religieuse de l'existence apaise mais implique un moment de rupture avec le monde extérieur. Ce sera le sens donné au périple et à l'illumination mystique vécue à Mende : l'homme est seul au monde face à la croix ; il ne peut chercher qu'en lui-même le sens de l'existence et s'assimiler le destin. La réécriture de l'Odyssée sur un mode kierkegaardien confère donc une cohérence à ces phases biographiques reconstituées par l'exilé.

Une cohérence aussi, si nous songeons au parcours biographique global de Döblin et à la très lente préparation que sa conversion avait présupposée : en effet, il serait erroné d'attribuer cet élan mystique à la seule urgence qu'il y avait en 1940 de donner un sens à la solitude et au dénuement de l'exilé. Bien d'autres textes contenaient déjà l'aveu d'une angoisse existentielle, celle qui s'était par exemple manifestée lors du voyage en Pologne et du choc que Döblin avait éprouvé en 1924 devant le crucifix de Veit Stoß à Cracovie ${ }^{19}$. Et si la conversion est l'apogée, l'ultime étape de son exil, c'est une manière de suggérer qu'un tel cheminement ne dépend pas de la seule initiative humaine mais qu'il est à imputer à une vocation spécifique, plus exactement à un destin individuel. Il est l'aboutissement d'un voyage compliqué et riche en revirements externes et internes, comme le titre de Schicksalsreise le rend intelligible.

danois, Leipzig, Lehmann, 1885, p. 453-627.

19. Helmuth Kiesel, Literarische Trauerarbeit. Das Exil- und Spätwerk Alfred Döblins, Tübingen, Niemeyer, 1986, p. 184-185. 


\section{Expériences d'un Robinson}

Le parrainage kierkegaardien justifierait également que la référence explicite ne soit pas l'Odyssée mais sa variante dans cet autre mythe qu'est « Robinson ». La première version de Schicksalsreise s'intitulait en effet « Robinson in Frankreich » et concernait la période précédant les retrouvailles de Döblin et de sa famille, sa femme et son fils. La formule qu'il y avait initialement placée en exergue a été conservée dans la publication de 1949 :

Diese Schrift widme ich meiner Frau Erna, die den schiffbrüchigen Robinson, wie man lesen wird, am Strand aufhob und ihn, dazu sich selbst und unsern Jüngsten rettete. Es war unser Schmerz, dass wir nicht noch andere, die uns am Herzen lagen, mitnehmen konnten. (p. 9)

Ce réemploi du mythe de Robinson sert à dissiper un risque de confusion entre mystique et mythe (ce qui, là encore, rapproche Döblin de Kierkegaard) : la vie spirituelle est de l'ordre de la foi, ses catégories ne sont pas à réduire à une pérégrination transitoire qui, elle, relève de la robinsonnade. Le mythe de Robinson rapproche du réel, des préoccupations matérielles, des solutions concrètes.

Il est réemployé pour trois de ses fonctions dans notre texte de Döblin. Celle du mythe de la survie. Celle aussi de l'apprentissage de la précarité et de la vie au stade originel, élémentaire («Ur-»). Elle fournit en outre le schéma narratif qui conduit à placer un personnage au centre du récit :

Es geschah mir in diesem Sommer 1940, daß ich wie Robinson auf den Strand einer fernen Insel im Weltmeere so in das Innere Frankreichs verschlagen wurde, ich bin in Mende im Flüchtlingslager. (p. 103)

En somme, cette parenté que Döblin instaure avec Robinson réinsère l'individu dans un système collectif, celui des exodiens ${ }^{20}$. Par ce truchement, auteur, narrateur, lecteur partagent une même connaissance des souffrances engendrées par l'isolement et le dénuement.

Mais Döblin a également tronqué le mythe de Robinson, l'a amputé, et les divergences sont importantes. Car le message du Robinson du XVIII ${ }^{\mathrm{e}}$ siècle était d'ordre moral, Defoe ayant fait triompher le puritanisme anglican : son Crusoe s'assagit et rentre dans le rang après avoir puisé son réconfort dans la lecture de la Bible $^{21}$. Nous sommes loin et du narrateur Döblin et des positions kierkegaardiennes en matière d'esthétique et d'éthique. Defoe, de surcroît, faisait l'apologie de la civilisation et du travail à partir des ressources matérielles dont regorge la nature, les progrès de la civilisation étaient censés garantir à cette génération le repos et la sécurité, alors que la situation s'est totalement inversée pour la génération de Döblin.

20. Ce néologisme regroupe toutes les catégories de personnes évacuées ou réfugiées; il se rencontre de plus en plus fréquemment, notamment depuis sa problématisation par Jean-Pierre Azéma dans 1940. L'année terrible, Paris, Seuil, 1990, p. 125, 128.

21. Dans l'article qu'il a dédié à Schicksalsreise dans Döblin-Handbuch: Leben - Werk - Wirkung, éd. par Sabina Becker, Stuttgart, Metzler, 2016, p. 303-311 (ici p. 306), Stefan Keppler-Kasaki va jusqu'à estimer qu'il y aurait aussi similitude au niveau religieux puisque Robinson Crusoe a un accès de délire religieux. 
Enfin, la question de la migration forcée se pose pour Robinson en termes de retour, comme chez Homère, alors que, dans Schicksalsreise, le nomadisme de l'âme reste le lot de l'humanité :

Baden-Baden. Ich bin am Ziel. Am Ziel, an welchem Ziel? (p. 308).

[...] Und wieder sehe ich: Ein Mensch hat es leichter als eine Stadt, sich zu ändern. Ein Mensch kann sich wandeln. Eine Stadt stürzt ein. (p. 348)

Döblin, même dans les pages relevant d'une robinsonnade, a brisé le confort rassurant que la lecture d'un mythe pourrait offrir. L'enchantement des fables peut se légitimer pour interpréter $u n$ moment de l'existence mais il ne peut plus fournir d'explication globale. La comparaison à Robinson n'est même pas dépourvue d'une dimension équivoque et ironique puisque le sauvetage est dû à une épouse plus pragmatique et mieux inspirée, autrement dit à une "Madame Robinson ${ }^{22}$ " qui privilégie la prise en compte de la dure réalité et dont les critères sont rationnels. Ce décalage est mis en exergue par l'insertion ${ }^{23}$, dans le récit de Döblin, du témoignage que sa femme a rédigé de sa propre évacuation et de son inquiétude, partagée par les autres civils, face à l'avancée des troupes allemandes : il s'agit d'une troisième forme d'écriture, qui se situe à mi-chemin entre celle d'un Stampfer et celle de Döblin. Elle se rapproche du premier par l'accent mis sur la sociabilité et par l'agencement chronologique et factuel, et du second par l'abondance de détails personnels et la proximité de sa relation avec le genre du journal intime, mais de parenté avec un mythe il ne saurait être question dans le compte rendu d'Erna Döblin.

La relation que Döblin a rédigée sur les événements de l'été 1940 occupe une place originale non seulement pour le témoignage qu'il a apporté de la désorganisation administrative, de la cartographie, de la sociologie des camps de réfugiés, mais aussi pour la compatibilité qu'il a instaurée entre mythographie et historiographie de l'exode. Il s'inscrit dans la tradition de l'Odyssée et de Robinson Crusoe, en conserve les caractéristiques de la mobilité et de ses contraintes, mais il remet en question l'espoir du retour dans la patrie et il le transpose sur un plan eschatologique. Schicksalsreise met ainsi en évidence la plurivocité de la réécriture du mythe chez Döblin, d'autant que d'autres mythes antiques y seraient d'ailleurs détectables, que ce soit celui du Christ en tant que contre-modèle de Prométhée ou encore celui de l'âme exilée dont la libération fut pensée par Platon. Döblin esquisse donc dans cet écrit les conditions qui aident le lecteur à s'approcher d'une pensée mythique : précarité matérielle, isolement, aspiration à la spiritualité, ascèse subie. Mais l'autonomie de l'œuvre littéraire n'en est pas pour autant entamée ou appauvrie car le recours à un moyen de dire ce que le langage courant ne suffit pas à exprimer a signifié sous sa plume que le mythe n'est ni seulement un objet littéraire ni la petite partie d'un patrimoine national mais un des éléments universels indispensables pour animer, élever et ouvrir la réflexion, qu'il répond à un besoin primordial de l'âme si

22. Thèse en cours d'Eva Rossignol-Raynal, L'expérience de l'aller et du retour dans Voyage et Destin et Hamlet ou la longue nuit prend fin (Alfred Döblin), L'Écriture ou la Vie et Le Grand Voyage (Jorge Semprun), Les Armes de la nuit et La Puissance du jour (Vercors).

23. « Eine andere Flucht», in Schicksalsreise, p. 175-202. 
elle aspire à s'élever au-dessus de la matière sans céder à la tentation de la mort, si bien que le destin et l'exil dépendent alors d'un libre choix. 
